\title{
POSITIVE HARMONIC FUNCTIONS OF A BRANCHING PROCESS
}

SERGE DUBUC

1. Introduction. Let $\{p(n)\}_{n=0}^{\infty}$ be a sequence of positive numbers, one defines an infinite matrix $p(x, y)(x, y) \in N \times N$ as follows:

$$
p(0, y)=\delta(0, y), \quad p(1, y)=p(y) ;
$$

the other lines of the matrix are such that

$$
p(x+1, y)=\sum_{y_{1}+y_{2}=y} p\left(1, y_{1}\right) p\left(x, y_{2}\right) .
$$

We will say that the matrix $p(x, y)$ is the branching matrix corresponding to the sequence $\{p(n)\}_{n=0}^{\infty}$. When $\sum_{n=0} p(n)=1$, the matrix is stochastic and describes a Markov process which is called a branching process.

We would like to get information about the positive harmonic functions $h(x)$ :

$$
\sum_{y} p(x, y) h(y)=h(x), \quad h(x) \geqq 0 .
$$

We will see that there is a one-to-one linear correspondence between these harmonic functions and those of a second triangular branching matrix. From that, it follows that there can be only one extreme harmonic function equal to 1 at $x=0$. This function whenever it exists is an exponential function. We will be able to describe all the harmonic functions only when $\sum_{x=0}^{\infty} p(n)=\sum_{n=0}^{\infty} n p(n)=1$. This case has been already solved by Kesten, Ney and Spitzer [1].

2. Functional meaning of harmonic functions. Let $h(x)$ be a positive function defined on $N$ and let $p(x, y)$ be the branching matrix corresponding to a sequence $\{p(n)\}_{n=0}^{\infty}$ of positive numbers, let us consider the cone of formal power series in a variable $z$ with positive coefficients. On this cone, we define the following functional:

$$
H(g(z))=\sum_{x=0}^{\infty} b(x) h(x) \quad \text { if } g(z)=\sum_{x=0}^{\infty} b(x) z^{x} .
$$

If $f(z)=\sum_{n=0}^{\infty} p(n) z^{n}$, we have that $(f(z))^{x}=\sum_{y=0}^{\infty} p(x, y) z^{y}$. Since $h(x)=H\left(z^{x}\right), h(x)$ is harmonic precisely when $H\left(z^{x}\right)=H((f(z)) x)$, that is to say when $H(g(z))=H(g(f(z)))$ for every power series $g(z)$.

Received by the editors March 11, 1968. 


\section{Transformation of harmonic functions.}

TheOREM. (a) If there is no number $a \geqq 0$ such that $\sum_{n-0}^{\infty} p(n) a^{n}$ converges to $a$, there is no harmonic function different from 0 .

(b) If $a$ is the smallest positive solution of $\sum_{n-0}^{\infty} p(n) t^{n}=t$, if $p^{\prime}(0)=0$ and

$$
p^{\prime}(n)=\sum_{m=-\infty}^{\infty}\left(\begin{array}{l}
m \\
n
\end{array}\right) p(m) a^{m-x} \quad(n \geqq 1),
$$

and if $p^{\prime}(x, y)$ is the branching matrix corresponding to the sequence $\left\{p^{\prime}(n)\right\}$, there is a linear one-to-one correspondence between positive harmonic functions of the matrix $p(x, y)$ and those of the matrix $p^{\prime}(x, y)$. Such a mapping is

$$
h^{\prime}(x)=\sum_{v=0}^{x}\left(\begin{array}{l}
x \\
v
\end{array}\right)(-a)^{x \rightarrow h} h(v), \quad h(x)=\sum_{v=0}^{x}\left(\begin{array}{l}
x \\
v
\end{array}\right) a^{x-v} h^{\prime}(v) .
$$

Proof. There is something to be proved only when $p(0) \neq 0$. Let $f_{n}(z)$ be the functional product of the power series $f(z) n$ times with itself. Let us start with a harmonic function $h(x)$ for the matrix $p(x, y)$. If $g(z)=\sum_{x=0}^{\infty} b(x) z^{x}$ is a power series such that $\sum_{x=0}^{\infty}|b(x)| h(x)<\infty$, we will set $H(g(z))=\sum_{x=0}^{\infty} b(x) h(x)$. Let $n$ be an integer and $t$ be a number between 0 and $f_{n}(0)$, we set $h_{t}(x)$ $=H\left((z-t)^{x}\right)$. Since $f_{n}(z)-t$ is a power series with positive coefficients, we get that $h_{t}(x)=H\left(\left(f_{\mathrm{n}}(z)-t\right)^{x}\right) \geqq 0$.

(a) If $\lim _{n \rightarrow \infty} f_{n}(0)=\infty$, then for every $t$,

$$
h_{t}(x)=\sum_{v=0}^{x}\left(\begin{array}{l}
x \\
v
\end{array}\right)(-t)^{x-v} h(v) \geqq 0 .
$$

So for every $x, h(x)=0$.

(b) If $\lim _{n \rightarrow \infty} f_{n}(0)<\infty, a=\lim _{n \rightarrow \infty} f_{n}(0)$. We will set $h^{\prime}(x)$ $=H\left((z-a)^{x}\right)$.

Since $h^{\prime}(x)=\lim _{t \uparrow a} h_{t}(x), h^{\prime}(x) \geqq 0$. On the cone of power series in a variable $w(w=z-a)$ with positive coefficients, $h^{\prime}(x)$ defines a positive functional $H^{\prime}$, where $H^{\prime}\left(w^{x}\right)=h^{\prime}(x)$.

$$
H^{\prime}\left((f(a+w)-a)^{x}\right)=H\left((f(z)-a)^{x}\right)=H\left((z-a)^{x}\right)=H^{\prime}\left(w^{x}\right) .
$$

Since $f(a+w)-f(a)=\sum_{n=0}^{\infty} p^{\prime}(n) w^{n}, k^{\prime}(x)$ is a harmonic function for the matrix $p^{\prime}(x, y)$.

Conversely, if $h^{\prime}(x)$ is a positive harmonic function for the matrix $p^{\prime}(x, y)$ and if $H^{\prime}$ is the corresponding functional on the power series in $w$, we set $h(x)=H^{\prime}\left((a+w)^{x}\right)$. From the change of variable $w \rightarrow f(a+w)-a$, which leaves $H^{\prime}$ unchanged, we get that $h(x)$ $=H^{\prime}\left((f(a+w))^{x}\right)$. So $h(x)$ is also a harmonic function and 


$$
h(x)=\sum_{v=0}^{x}\left(\begin{array}{l}
x \\
v
\end{array}\right) a^{y-v} h^{\prime}(v) .
$$

4. Applications. Let us remark that the matrix $p^{\prime}(x, y)$ is triangular: if $y<x, p^{\prime}(x, y)=0$. On the diagonal $p^{\prime}(x, x)=\mu^{x}$ where $\mu=\sum_{1}^{\infty} m p(m) a^{m-1} . \mu$ is the derivative of $f(z)$ at $z=a$, so $\mu \leqq 1$. If $h^{\prime}(x)$ is an extreme function such that $h^{\prime}(0)=1$, since $\delta(0, x)$ is harmonic and from the fact that $h^{\prime}(x)=\left[h^{\prime}(x)-\delta(0, x)\right]+\delta(0, x)$ is the sum of two positive harmonic functions, then $h^{\prime}(x)=\delta(0, x)$. The corresponding harmonic function $h(x)$ is $a^{x}$. This exponential is the only extreme harmonic function taking the value 1 at $x=0$ for the matrix $p(x, y)$. This last fact could have been proven directly. Let us sketch two other proofs for that.

Let $h(x)$ be an extreme harmonic function for $p(x, y)$ such that $h(0)=1$.

(a) Let $\tilde{h}(x)=\min \left\{h\left(y_{1}\right) h\left(y_{2}\right) \cdots h\left(y_{x}\right) \mid y_{1}+y_{2}+\cdots+y_{x}=x\right\}$; we will check that $\tilde{h}(x)$ is a superharmonic function. Let $w_{1}, w_{2}, \cdots$, $w_{x}$ be $x$ nonnegative integers whose sum is $x$, then

$$
\begin{aligned}
\sum_{y} p(x, y) \tilde{h}(y) \\
\quad=\sum_{z_{1}, z_{2}, \cdots, z_{z}} p\left(w_{1}, z_{1}\right) p\left(w_{2}, z_{2}\right) \cdots p\left(w_{x}, z_{x}\right) \tilde{h}\left(z_{1}+z_{2}+\cdots+z_{x}\right) \\
\quad \leqq \sum_{z_{1}, z_{2}, \cdots, z_{x}} p\left(w_{1}, z_{1}\right) \cdots p\left(w_{x}, z_{x}\right) h\left(z_{1}\right) \cdots h\left(z_{x}\right) \\
\quad \leqq h\left(w_{1}\right) h\left(w_{2}\right) \cdots h\left(w_{x}\right) .
\end{aligned}
$$

Hence $\sum_{\nu} p(x, y) \tilde{h}(y) \leqq \tilde{h}(x)$.

Using the Riesz decomposition theorem for superharmonic function and noticing that in our case there is no potential $U(x)$ such that $U(0)=1$, we get that $\tilde{h}(x)$ is harmonic and is equal to $h(x)$.

(b) $\delta(0, x)$ is a subharmonic function which is smaller than $h(x)$, so $h(x)=\lim _{n \rightarrow \infty} \sum_{y=0}^{\infty} p_{n}(x, y) \delta(0, y)=\lim _{n \rightarrow \infty}\left(f_{n}(0)\right)^{x}$ where $p_{n}(x, y)$ is the branching process corresponding to the power series $f_{n}(z)$.

If $\mu=1$ and if $h^{\prime}(x)$ is a harmonic function such that $h^{\prime}(1)=0$, one can check easily that $h^{\prime}(x)=0$ if $x>0$. So the only positive harmonic functions are in that case $A \delta(0, x)+B \delta(1, x)$ where $A \geqq 0, B \geqq 0$.

\section{REFERENCE}

1. H. Kesten, P. Ney and F. Spitzer, The Galton-Watson process with mean one and finite variance, Teor. Verojatnost. i Primenen. 11 (1966), 579-611.

Universite de Montréal 